\title{
AVALIAÇÃO DA CAPACIDADE DE ABSORÇÃO DE ÁGUA DE INGREDIENTES E ADITIVOS UTILIZADOS NA INDÚSTRIA DE CARNES SUBMETIDOS A DIFERENTES CONCENTRAÇÕES SALINAS
}

KÖHN, C.R. ${ }^{1}$; KEMPKA, A. P. ${ }^{2}$ e PRESTES, R.C. ${ }^{3}$

${ }^{1}$ Universidade Federal de Santa Maria, Departamento de Tecnologia e Ciência dos Alimentos

${ }^{2}$ Universidade do Estado de Santa Catarina, Departamento de Engenharia de Alimentos

3* Universidade Federal de Santa Maria, Departamento de Tecnologia e Ciência dos Alimentos

*E-mail para contato: rosacrisprestes@ @otmail.com

\begin{abstract}
RESUMO - A capacidade de absorção de água (CAA) é uma propriedade economicamente importante porque afeta o rendimento e a qualidade dos produtos cárneos. Este estudo avaliou a CAA $(\%)$ de treze amostras de ingredientes e aditivos divididos em dois grupos (proteínas e polissacarídeos) através da adição de soluções salinas com diferentes concentrações de cloreto de sódio $(2 \%, 4 \%$, $6 \%$ e $8 \%$ ), visando à simulação das condições de utilização em salmouras industriais. $\mathrm{O}$ método consistiu na adição da solução, centrifugação, secagem e pesagem. Houve diferença significativa $(\mathrm{p}<0,05)$ nos valores da CAA $(\%)$ para cinco das treze amostras avaliadas e também houve diferença significativa ( $\mathrm{p}<$ $0,05)$ nas diferentes concentrações salinas. A maior CAA $(\%)$ foi encontrada para goma guar $(639,96 \%$ a $8 \%)$ e menor para a fécula de mandioca $(67,08 \%$ a $2 \%)$. No grupo das proteínas os maiores e menores valores foram $368,26 \%$ a $8 \%$ e $187,95 \%$ a $2 \%$ para proteína concentrada de soja e proteína texturizada de soja, respectivamente.
\end{abstract}

\section{INTRODUÇÃO}

A capacidade de absorção de água (CAA) ou capacidade de retenção de água (CRA) consiste na adição de água ou de uma solução aquosa ao material com posterior centrifugação e quantificação da água que ficou retida pelo material sedimentado no tubo da centrífuga (Damoradan et al., 2010). A CAA é economicamente importante 
para a indústria processadora de carne, pois a perda de umidade afetará negativamente o rendimento e os atributos de qualidade dos produtos (Ordóñez et al., 2005). Segundo Wang et al. (2006) altos valores de capacidade de absorção de água são importantes para ajudar a manter a umidade dos produtos.

Diferentes metodologias para avaliação da CAA são descritas na literatura (Barbut, 1996; Wang et al., 2006; Tsai et al., 1998; Hedenus et al., 2000). Porém, existe uma lacuna na literatura em relação a estudos que avaliem a CAA dos ingredientes e aditivos quando estes forem submetidos a diferentes concentrações salinas ou em salmouras que são as formas mais comuns de utilização ou veiculação dos ingredientes e aditivos nos produtos industrializados pelas indústrias processadoras. Também existe a necessidade de gerar informação para as indústrias com objetivo de auxiliar na escolha dos ingredientes e aditivos no desenvolvimento de novas formulações dos produtos cárneos.

Neste contexto, o objetivo deste estudo foi avaliar e comparar a CAA (\%) de vários ingredientes e aditivos utilizados na indústria de carnes submetidos a diferentes concentrações salinas.

\section{MATERIAIS E MÉTODOS}

Os experimentos foram realizados no Laboratório de Físico-Química do Departamento de Tecnologia e Ciência dos Alimentos (DTCA) da Universidade Federal de Santa Maria (UFSM) em Santa Maria (RS). Os ingredientes e aditivos foram agrupados em dois grupos (proteínas e polissacarídeos) e foram testados: (PIS) proteína isolada de soja (ADM, São Paulo, SP), (PTS1) proteína texturizada de soja (Doremus, Guarulhos, SP), (PTS2) proteína texturizada de soja (Marsul, Montenegro, RS), (PCS) proteína concentrada de soja (Marsul, Montenegro, RS), (GG) goma guar (Nutract, Chapecó, SC), (CAR) kappa-carragena (Doremus, Guarulhos, SP), (AU8) amido modificado Ultratex-8 (National Starch, Jaguaré, SP), (AN) amido modificado Novation 1900 (National Starch, Jaguaré, SP), (AE) amido modificado Eliane 100 (National Starch, Jaguaré, SP), (AT) amido modificado Thermetex (National Starch, Jaguaré, SP), (AMN) amido modificado Novation 2300/EK 8925 (National Starch, Jaguaré, SP), (FM) fécula de mandioca (Lar, Medianeira, PR) e (AU3) amido modificado Ultratex 3 (National Starch, Jaguaré, SP). 
Para avaliação da CAA (\%) o método consistiu na pesagem de 1,56 g de amostra para cada tubo de centrífuga (tubos plásticos tipo Falcon com capacidade de $15 \mathrm{~mL}$ ) e adicionados 9,6 mL de solução salina. Para o preparo das soluções foi utilizado cloreto de sódio (Sigma) nas concentrações de $2 \%, 4 \%, 6 \%$ e $8 \%$. Após a adição da solução foi realizada a agitação manual do tubo por aproximadamente 1 minuto. Logo após, os tubos foram colocados em repouso por 10 minutos e posteriormente foram centrifugados durante 25 minutos a 2.900 g (centrífuga ITR, modelo super II, Instrumentos para Laboratórios LTDA, Esteio, RS). O sobrenadante foi descartado e os tubos foram submetidos à secagem em estufa com circulação de ar a $50{ }^{\circ} \mathrm{C}$ por 20 minutos com inclinação para baixo de $15^{\circ}$ a $20^{\circ}$ (Estufa Marconi, modelo MA033, Piracicaba, SP). Após a secagem, os tubos foram novamente pesados e calculou-se a CAA (\%) para cada amostra levando-se em consideração a diferença de peso conforme metodologia modificada de Sosulski (1962) citada por Wang et al. (2006).

O delineamento experimental foi inteiramente casualizado e foram realizadas três repetições para cada teste e para cada ingrediente ou aditivo. Os resultados foram submetidos à Análise de Variância - ANOVA e Teste de Tukey com nível de significância de $95 \%$ (p< 0,05) utilizando o programa Statistica ${ }^{\circledR} 9.0$ (STATSOFT, INC).

\section{RESULTADOS E DISCUSSÃO}

Na Tabela 1 estão apresentados os resultados obtidos para a CAA (\%) para cada ingrediente ou aditivo avaliado nas diferentes concentrações de solução salina (2, 4, 6 e $8 \%)$. Analisando a tabela observa-se que houve diferença significativa $(\mathrm{p}<0,05)$ nos valores da CAA $(\%)$ entre os ingredientes e aditivos testados e também houve diferença significativa $(\mathrm{p}<0,05)$ nas diferentes concentrações salinas, exceto a PIS que não apresentou variação significativa na CAA com o aumento da concentração salina da solução adicionada.

Verificou-se que os amidos modificados AE, AT e a fécula de mandioca (FM) apresentaram os menores valores de CAA e que também houve diferença significativa $(\mathrm{p}<0,05)$ para diferentes proteínas de soja testadas. As baixas CAA para a FM podem ser justificadas devido aos grânulos de amido serem insolúveis e se hidratarem muito pouco em água fria (Damoradan et al., 2010). 
Tabela 1 - Capacidade de Absorção de Água (CAA) em \% para os ingredientes ou aditivos avaliados (proteínas e polissacarídeos) nas diferentes concentrações salinas testadas

\begin{tabular}{|c|c|c|c|c|c|}
\hline \multicolumn{2}{|c|}{$\begin{array}{l}\text { Grupos/Concentrações } \\
\text { salinas* }\end{array}$} & $2 \%$ & $4 \%$ & $6 \%$ & $8 \%$ \\
\hline \multirow{4}{*}{ Proteínas } & PIS & $302,75^{\mathrm{gA}} \pm 1,50$ & $317,87^{\mathrm{gA}} \pm 8,27$ & $313,32^{\mathrm{gA}} \pm 4,89$ & $316,91^{\mathrm{gA}} \pm 1,84$ \\
\hline & PTS1 & $187,96^{\mathrm{eA}} \pm 2,68$ & $220,67^{\mathrm{dB}} \pm 4,43$ & $190,89^{\mathrm{eA}} \pm 2,31$ & $209,97^{\mathrm{eB}} \pm 8,23$ \\
\hline & PTS2 & $238,43^{\mathrm{fAB}} \pm 8,09$ & $293,28^{\mathrm{fB}} \pm 2,75$ & $194,06^{\mathrm{eA}} \pm 3,57$ & $212,00^{\mathrm{eAB}} \pm 8,09$ \\
\hline & PCS & $315,66^{\mathrm{hB}} \pm 4,61$ & $265,18^{\mathrm{eA}} \pm 9,06$ & $254,82^{\mathrm{fA}} \pm 3,47$ & $368,26^{\mathrm{hC}} \pm 3,23$ \\
\hline \multirow{9}{*}{ Polissacarídeos } & $\mathrm{AT}$ & $87,05^{\mathrm{bA}} \pm 0,67$ & $87,31^{\mathrm{a}} \mathrm{A} \pm 0,99$ & $89,90^{\mathrm{bB}} \pm 0,06$ & $85,80^{\mathrm{bA}} \pm 0,14$ \\
\hline & AU8 & $332,84^{\mathrm{iD}} \pm 0,46$ & $272,85^{\mathrm{eC}} \pm 0,96$ & $264,02^{\mathrm{fB}} \pm 1,43$ & $259,47^{\mathrm{fA}} \pm 0,32$ \\
\hline & AU3 & $512,16^{\mathrm{jB}} \pm 3,18$ & $513,51^{\mathrm{hB}} \pm 5,13$ & $194,51^{\mathrm{eA}} \pm 4,17$ & $515,32^{\mathrm{iB}} \pm 4,91$ \\
\hline & $\mathrm{AE}$ & $86,05^{\mathrm{bC}} \pm 0,74$ & $81,36^{\mathrm{aAB}} \pm 0,84$ & $79,63^{\mathrm{abA}} \pm 0,42$ & $83,46^{\mathrm{abB}} \pm 0,43$ \\
\hline & AMN & $112,15^{\mathrm{cB}} \pm 0,49$ & $106,04^{\mathrm{bA}} \pm 1,60$ & $125,49^{\mathrm{cC}} \pm 0,01$ & $107,49^{\mathrm{cA}} \pm 1,28$ \\
\hline & AN & $115,14^{\mathrm{cA}} \pm 3,41$ & $125,33^{\mathrm{cB}} \pm 1,64$ & $121,68^{\mathrm{cAB}} \pm 1,62$ & $122,18^{\mathrm{cAB}} \pm 0,17$ \\
\hline & CAR & $166,08^{\mathrm{dA}} \pm 0,10$ & $210,74^{\mathrm{dC}} \pm 3,44$ & $151,71^{\mathrm{dA}} \pm 0,48$ & $193,32^{\mathrm{dA}} \pm 7,53$ \\
\hline & GG & $599,47^{\mathrm{kA}} \pm 1,23$ & $613,60^{\mathrm{iB}} \pm 0,43$ & $630,64^{\mathrm{hC}} \pm 0,29$ & $639,97^{\mathrm{j} D} \pm 1,60$ \\
\hline & FM & $67,08^{\mathrm{aA}} \pm 0,23$ & $74,19^{\mathrm{a} C} \pm 0,50$ & $72,72^{\mathrm{aAB}} \pm 0,13$ & $70,74^{\mathrm{aB}} \pm 1,44$ \\
\hline
\end{tabular}

* Médias com letras minúsculas diferentes na vertical diferem significativamente ( $\mathrm{p}<$ $0,05)$.

** Médias com letras maiúsculas na horizontal diferem significativamente $(\mathrm{p}<0,05)$.

*** As siglas correspondem a: (PIS) proteína isolada de soja, (PTS1) proteína texturizada de soja 1, (PTS2) proteína texturizada de soja 2, (GG) goma guar, (PCS) proteína concentrada de soja, (CAR) kappa-carragena, (AU8) amido modificado Ultratex-8, (AN) amido modificado Novation 1900, (AE) amido modificado Eliane 100, (AT) amido modificado Thermetex, (AMN) amido modificado Novation 2300/EK 8925, (FM) fécula de Mandioca e (AU3) amido modificado Ultratex 3. 
A adição de sais às soluções polissacarídicas pode promover modificações, aumentando ou diminuindo a solubilidade. A variação no grau dos substituintes de um polissacarídeo altera suas propriedades reológicas em solução, cujo comportamento pode ser atribuído à interação entre resíduos carregados e íons provenientes da dissociação do sal, quando presentes em solução. Este comportamento pode indicar que uma cadeia mais distendida favorece a solubilidade e conduz a uma menor viscosidade, pois a densidade de cargas (número de cargas por unidade de comprimento) modifica as propriedades macromoleculares em solução (Aranda-Selverio et al., 2010). Ramaswamy et al. (2013) mencionaram que cadeias de arabinanas com conformação linear apresentaram maior capacidade de retenção de água do que as cadeias ramificadas o que pode ser explicado pela maior mobilidade da molécula em solução permitindo maior interação com a água.

A adição do sal pode levar à aproximação do PI (ponto isoelétrico) das proteínas e isto altera a solubilidade das proteínas, pois modifica a distribuição de aminoácidos hidrofóbicos e hidrofílicos na superfície da proteína (Araújo, 2008). O comportamento de soluções proteicas é acentuadamente afetado pela presença de íons de baixa massa molar especialmente os ânions e cátions presentes nos sais. O cloreto de sódio em determinadas concentrações acentua a solubilidade das proteínas. Ocorre o mecanismo denominado salting-in onde os grupos carregados na superfície atraem e ligam ânions e cátions mais fortemente do que fazem com a água. No entanto, esses íons ainda trazem junto de si um agrupamento ordenado das suas próprias moléculas de água de solvatação, as quais mantêm a molécula de proteína em solução. Concentrações mais elevadas de eletrólitos promovem a precipitação das proteínas em solução, fenômeno conhecido como salting-out. Há uma competição dos íons do sal com a proteína pela água necessária a fim de manter a proteína em solução de forma que, se a concentração salina se eleva as proteínas precipitam (Coultate, 2004).

Analisando-se as proteínas de soja, os maiores valores de CAA (\%) foram encontrados para PCS na concentração de $8 \%(368,26 \%)$ o que pode ser justificado pela maior área de superfície ocasionada pela menor granulometria (pó) em relação aos outros tipos de proteína (flocos ou grânulos) e também pela maior solubilidade desta proteína decorrente do processo de purificação e obtenção ao nível industrial. As proteínas PTS1 e PTS2 diferiram na CAA na concentração salina de $2 \%$ e $4 \%$. Essa diferença pode ser explicada pela granulometria, teor de proteínas e possíveis variações no processo de obtenção destas proteínas pelos fabricantes. 
A maior CAA para AU3 pode ser explicada pela interação entre resíduos carregados e íons provenientes da dissociação do sal, quando presentes em solução proporcionando uma cadeia mais distendida o que favorece a solubilidade conforme sugeriram Aranda-Selverio et al. (2010).

A GG e PIS apresentaram a maior CAA nos diferentes grupos para as quatro diferentes soluções salinas, sendo que a GG apresentou o maior valor de absorção de água entre todos os ingredientes e aditivos testados chegando até 639,97\% (cerca de 6,4 vezes o seu peso em água). Os maiores valores de CAA $(\%)$ foram encontrados para goma guar (GG) $(639,97 \%)$, amido modificado (AU3) $(515,32)$ e, proteína concentrada de soja (PCS) $(368,26)$.

\section{CONCLUSÃO}

Conclui-se que houve variação significativa $(\mathrm{p}<0,05)$ na CAA $(\%)$ para os diferentes grupos de aditivos e ingredientes testados e que para quatro amostras a maior absorção foi obtida na concentração salina de $8 \%$. Não houve um comportamento homogêneo entre os grupos de polissacarídeos e proteínas avaliadas e mesmo para um determinado tipo de proteína houve variação entre as amostras testadas conforme a adição das diferentes soluções. Os resultados encontrados podem auxiliar no desenvolvimento de novas formulações de produtos cárneos a nível industrial, diminuindo tempo de testes e facilitando a escolha do melhor ou mais adequado ingrediente/aditivo de acordo com a aplicação e/ou concentração salina no produto final.

\section{REFERÊNCIAS BIBLIOGRÁFICAS}

ARANDA-SELVERIO, G.; PENNA, A.L.B.; CAMPOS-SÁS, L.F.; SANTOS JUNIOR, O.S.; VASCONCELOS, A.F.D.; SILVA, M.L.C.,; LEMOS, E.G.M.,; CAMPANHARO, J.C.; SILVEIRA J.L.M. Propriedades reológicas e efeito da 
adição de sal na viscosidade de expolissacarídeos produzidos por bactérias do gênero Rhizobium. Química Nova, v. 33, n. 4, p. 895-899, 2010.

ARAÚJO, J.M.A. Química de alimentos: teoria e prática. Viçosa: UFV, 596p, 2008.

BARBUT, S. Estimates and detection of the PSE problem in young turkey breast meat. Canadian Journal of Animal Science, v.76, p. 455-457, 1996.

COULTATE, T.P. Alimentos: a química de seus componentes. Porto Alegre: Artmed, 368p, 2004.

DAMORADAN, S.; PARKIN, K.L.; FENNEMA, O. Química de Alimentos de Fennema. Porto Alegre: Artmed, 900p, 2010.

HEDENUS, P.; MATTSSON, M.S.; NIKLASSON, G.A.; CAMBER, O.; EK, R. Characterization of instantaneous water absorption properties of pharmaceutical excipients. International Journal of Pharmaceutics, v.202, n.1-2, p.141-149, 2000.

ORDÓÑEZ, J.A. Tecnologia de Alimentos. Porto Alegre: Artmed, 279p, 2005.

RAMASWAMY, U.; KABEL, M.A.; SCHOLS, H.; GRUPPEN, H. Structural features and water holding capacities of pressed potato fibre polysaccharides. Carbohydrate Polymers, v. 93, n.2, p.589-596, 2013.

WANG, S.H.; ROCHA, G.O.; NASCIMENTO, T.; ASCHERI, J.L.R. Absorção de água e propriedades espumantes de farinhas extrusadas de trigo e soja. Ciência e Tecnologia em Alimentos, v.26, n.2, p.475-481, 2006.

TSAI, S.; UNKLESBAY, N.; UNKLESBAY, K.; CLARKE, A. Water and absorptive properties of restructured beef with five binders of four isothermal temperatures. Food Science Technology, v.31. n.1., p.78-83, 1998. 